\title{
Regrowth of stellar disks in mature galaxies: The two component nature of NGC 7217 revisited with VIRUS- $W^{\dagger, \diamond}$
}

\author{
Maximilian H. Fabricius, ${ }^{1,2}$ Lodovico Coccato,${ }^{3}$ Ralf Bender, ${ }^{1,2}$ \\ Niv Drory, ${ }^{4}$ Claus Gössl, ${ }^{2}$ Martin Landriau, ${ }^{4}$ Roberto P. Saglia, ${ }^{1}$ \\ Jens Thomas, ${ }^{1}$ and Michael J. Williams ${ }^{1,5}$ \\ ${ }^{1}$ Max Planck Institute for Extraterrestrial Physics, Giessenbachstraße, 85748 Garching, \\ Germany \\ ${ }^{2}$ University Observatory Munich, Scheinerstraße 1, 81679 Munich, Germany \\ ${ }^{3}$ European Southern Observatory, Karl-Schwarzschild-Straße 2, D-85748 Garching bei \\ Muenchen, Germany \\ ${ }^{4}$ McDonald Observatory, The University of Texas at Austin, 2515 Speedway, Stop C1402, \\ Austin, Texas 78712-1206, USA \\ ${ }^{5}$ Department of Astronomy, Columbia University, New York 10027, USA
}

\begin{abstract}
We have obtained high spectral resolution $(R \approx 9000)$, integral field observations of the three spiral galaxies NGC 3521, NGC 7217 and NGC 7331 using the new fiber-based Integral Field Unit instrument VIRUS-W at the $2.7 \mathrm{~m}$ telescope of the McDonald Observatory in Texas. Our data allow us to revisit previous claims of counter rotation in these objects. A detailed kinematic decomposition of NGC 7217 shows that no counter rotating stellar component is present. We find that NGC 7217 hosts a low dispersion, rotating disk that is embedded in a high velocity dispersion stellar halo or bulge that is co-rotating with the disk. Due to the very different velocity dispersions $\left(\approx 20 \mathrm{~km} \mathrm{~s}^{-1}\right.$ vs. $\left.150 \mathrm{~km} \mathrm{~s}^{-1}\right)$, we are further able to perform a Lick index analysis on both components separately which indicates that the two stellar populations are clearly separated in $(\mathrm{Mg} b,\langle\mathrm{Fe}\rangle)$ space. The velocities and dispersions of the faster component are very similar to those of the interstellar gas as measured from the $[\mathrm{O}$ III] emission. Morphological evidence of active star formation in this component further suggests that NGC 7217 may be in the process of (re)growing a disk inside a more massive and higher dispersion stellar halo.
\end{abstract}

Keywords. galaxies: bulges — galaxies: evolution — galaxies: formation — galaxies: structure ${ }^{\dagger}$ This paper includes data taken at The McDonald Observatory of The University of Texas at Austin.

${ }^{\diamond}$ This paper contains data obtained at the Wendelstein Observatory of the Ludwig-Maximilians University Munich.

\section{Introduction}

The decomposition of galaxies into the structural components such as bulges, disks and bars is an essential tool to help the understanding of the formation of these systems. For a long time this has been limited to photometry alone, either through the decomposition of surface brightness profiles or the direct modelling of 2D images (e.g. Peng et al. 2002). More recently several approaches have been taken to decompose also spectra into multiple components (e.g. Vergani et al. 2007; Coccato et al. 2011; Johnston et al. 2012; Coccato et al. 2013, see also the parallel contribution by Coccato et al.). Some of these methods make explicitly use of the different kinematics of for instance two counter rotating components. 

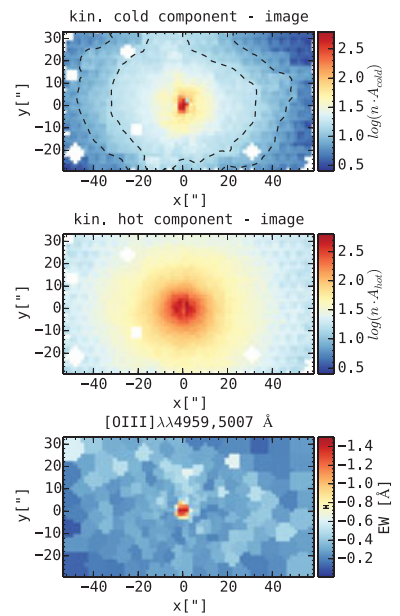
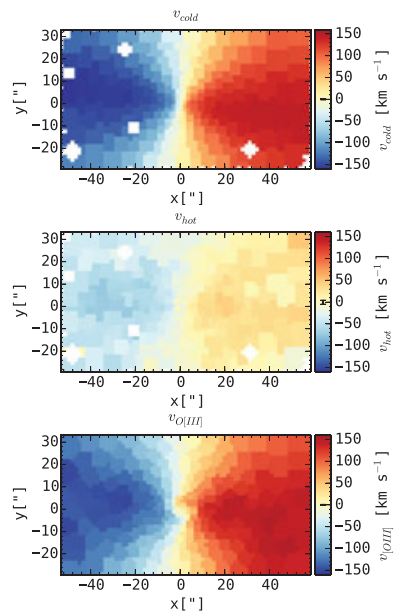
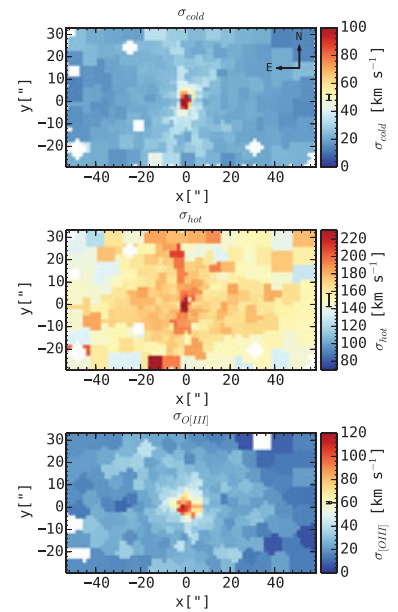

Figure 1. Two-dimensional maps of the of the stellar kinematics obtained from the doubleGaussian decomposition and the gas kinematics. We plot from left to right: the amplitude, the mean velocity and the velocity dispersion of the best fitting model. The upper row plots the moments for the cold component while the middle row plots the hot component. The amplitudes for the two components have been normalized such that their integrated sum equals the wavelength collapsed datacube. To highlight the flattening of the cold disk component, we added contours at two arbitrary values. The bottom row shows the summed equivalent width of the $[\mathrm{O}$ III $] \lambda \lambda$ 4959,5007 $\AA$ emission lines and the velocity and velocity dispersion obtained by the kinematics extraction routine from the brighter line at $5007 \AA$. In all maps positive $y$-values point to north and positive $x$-values to west. Reproduced from Fabricius et al. 2014.

While the spectral decomposition requires high signal to noise (SN) spectra, a large spectral resolution, and consequently large integration times, it has two significant advantages over the photometric decomposition: First, it is model free, it does not rely on the inwards extrapolation of an exponential profile for instance. Secondly, it allows us to study absorption line strengths of the superimposed components. Thus ages, metallicities and over-abundances of the stellar components can be studied separately.

NGC 7217 was previously claimed to host two counter rotating stellar disks (Merrifield \& Kuijken 1994) based on double peaked line of sight velocity distributions (LOSVDs) and high velocity tails. This has been questioned by Buta et al. 1995 (hereafter B95) who claims that at least the tails can be explained by a massive but low surface brightness stellar halo. Using the new Integral Field Unit (IFU) spectrograph VIRUS-W we have obtained spectra that we analyse using a new algorithm to derive non-parametric LOSVDs but also an established algorithm to derive parametric two component models.

All results have been presented in Fabricius et al. (2014). Here we give a brief summary of our observations, the analysis techniques and our findings.

\section{Data \& Analysis}

We observed NGC 3521, NGC 7217 and NGC 7331 using the fiber based Integral-Field Unit spectrograph (IFU) VIRUS-W at the $2.7 \mathrm{~m}$ (Fabricius et al. 2012) telescope of the McDonald Observatory in Texas. We used the stellar dynamics mode of the instrument that offers a spectral resolution of $R \approx 9000\left(\sigma_{\text {inst }}=15 \mathrm{~km} \mathrm{~s}^{-1}\right)$ in the optical. The IFU has a total field of view of $105 \operatorname{arcsec} \times 55$ arcsec with a fill factor of $1 / 3$ such that three dithered observations are needed to fill the field of view completely. We interleaved dithers with sky nods for background subtraction. The observations were carried out in 


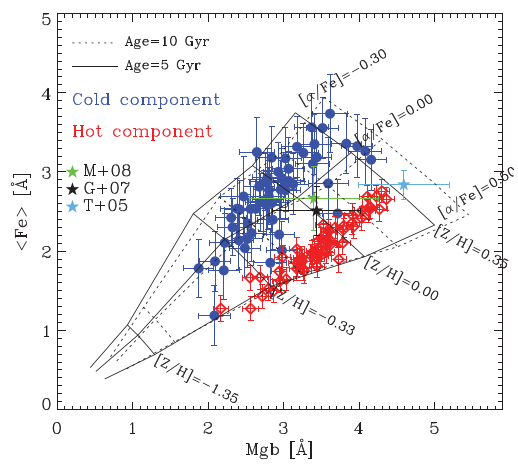

Figure 2. $\mathrm{Mg} b$ and $\langle\mathrm{Fe}\rangle$ line strengths indices measured for the cold component (blue circles) and the hot component (red diamonds) in NGC 7217. Predictions from single stellar population models (Thomas et al. 2003) are also shown for comparison. The black, and green stars represent the mean values of the measurements by Gorgas et al. (2007) and Morelli et al. (2008) for a sample of bulges in spiral galaxies with the corresponding error bars representing the standard deviations of their measurements. The light blue star and its error bars represents the mean measurements and the standard deviation of a sample of early-type galaxies by Thomas et al. (2005). Reproduced from Fabricius et al. 2014.

dark time in August 2011, May 2011 and June 2012. Individual on-object integration times were limited to $1800 \mathrm{~s}$ to sample the sky regularly. The total on-object integration time per fiber amounts to $1.2 \mathrm{~h}, 1.5 \mathrm{~h}$ and $4.5 \mathrm{~h}$ for NGC 3521, NGC 7217 and NGC 7331 respectively.

For the primary kinematic extraction we employ a new version of the Maximum Penalized Likelihood which adds the treatment of nebular emission lines. This method allows us to derive non-parametric line-of-sight velocity distributions that show an obvious double component structure. We decompose the LOSVDs using two Gaussian models. In a second step we use the parametric spectral decomposition technique of Coccato et al. (2011) to confirm the result and to derive line strength indices for the two different components separately.

\section{Results}

In Fig. 1 we show the relative weights, the velocities and the velocity dispersions of the two different stellar components in NGC 7217. We also show the equivalent width, and the velocity and the velocity dispersion of the nebular emission as measured from the [O III] lines. We find that rather than two counter rotating stellar disks, NGC 7217 hosts a low dispersion $\left(\approx 20 \mathrm{~km} \mathrm{~s}^{-1}\right)$, rotating $\left(v_{\max }=150 \mathrm{~km} \mathrm{~s}^{-1}\right)$ disk that is embedded in a high velocity dispersion stellar halo $\left(\approx 170 \mathrm{~km} \mathrm{~s}^{-1}\right)$ or bulge that is co-rotating $\left(v_{\max }=50 \mathrm{~km} \mathrm{~s}^{-1}\right)$ with the disk. With the exception of the central arcseconds the low dispersion disk appears well aligned with the gas disk. Both the velocity fields and the dispersions are very similar, suggesting that if gas disk is a residual to the formation of the stellar disk, then the stellar disk is yet dynamically young. A structural analysis of the relative weights of the two gaussian components as function of position shows great similarity with the photometric decomposition by B95. The low dispersion component therefore is very likely identical to the exponential disk by B95 while the high dispersion halo represents his de Vaucouleurs' bulge (Fabricius et al. 2014).

The parametric spectral decomposition reveals that the lower dispersion component forms essentially a parallel sequence to the high dispersion component in the $(\mathrm{Mg} b,\langle\mathrm{Fe}\rangle)$ absorption line strength diagram (see Fig. 3). Both sequences span a range of about 2 $\AA$ both in $\mathrm{Fe}$ and $\mathrm{Mg} b$ but the disk is offset by about an $\AA$ towards either larger Fe strengths, lower $\mathrm{Mg} b$ strengths or a combination of both.

\section{Conclusions}

Using high SN and large spectral resolution optical IFU data we have derived velocity and velocity dispersion maps for two separate, superimposed stellar components in NGC 
7217. We were able to show that those correspond to a dominant, high velocity dispersion and slowly rotating stellar halo and an embedded, rotating stellar disk. The disk only contains about $30 \%$ of the total light. Given its bluer color and the obvious sites of active star formation in imaging data it almost certainly has a lower mass to light ratio than the halo. Its mass fraction is therefore even lower that $30 \%$. The comparison of the line strengths with single stellar population models (Thomas et al. 2003) in the $(\mathrm{Mg} b,\langle\mathrm{Fe}\rangle)$ plane shows that the disk component is offset to lower $[\alpha / \mathrm{Fe}]$ over-abundances but does not extend to higher metallicities than the high dispersion halo. This would be naturally explained by an accretion of relatively primordial material, that restarted the star formation at much lower metallicities but at the maximum over-abundance of the high dispersion component. The photometric and spectroscopic properties of the halo component are in several aspects more similar to an elliptical galaxy than to those of the bulges of spiral galaxies. If the halo is merger-built, than the disk would not have survived the merging event. We suggest that it must have formed after the merger, either from material that remained at large radii throughout the time of coalescence or from material that was accreted later through minor mergers or cold accretion from the intergalactic medium (Mazzuca et al. 2006; Eliche-Moral et al. 2010).

We are currently in the process of analysing the data for NGC 3521 and NGC 7331 that have similar previous claims of counter rotation Prada et al. (1996); Zeilinger et al. (2001).

\section{Acknowledgements}

We would like to thank the organizers for this wonderful conference. L. C. acknowledges financial support from the European Communitys Seventh Framework Program (/FP7/2007-2013/) under grant agreement No. 229517. This research has made use of the NASA/IPAC Extragalactic Database (NED) which is operated by the Jet Propulsion Laboratory, California Institute of Technology, under contract with the National Aeronautics and Space Administration.

\section{References}

Buta, R., van Driel, W., Braine, J., et al. 1995, ApJ, 450, 593

Coccato, L., Morelli, L., Corsini, E. M., et al. 2011, MNRAS, 412, L113

Coccato, L., Morelli, L., Pizzella, A., et al. 2013, A\&A, 549, A3

Eliche-Moral, M. C., González-García, A. C., Balcells, M., et al. 2010, in AIP Conference Series, Vol. 1240, ed. V. P. Debattista \& C. C. Popescu, 237-238

Fabricius, M. H., Coccato, L., \& Bender, R. 2014, MNRAS, 441, 2212

Fabricius, M. H., Grupp, F., Bender, R., et al. 2012, in SPIE Conference Series, Vol. 8446

Gorgas, J., Jablonka, P., \& Goudfrooij, P. 2007, A\&A, 474, 1081

Johnston, E. J., Aragón-Salamanca, A., Merrifield, M. R., \& Bedregal, A. G. 2012, MNRAS, 422,2590

Mazzuca, L. M., Sarzi, M., Knapen, J. H., Veilleux, S., \& Swaters, R. 2006, ApJ, 649, L79

Merrifield, M. R. \& Kuijken, K. 1994, ApJ, 432, 575

Morelli, L., Pompei, E., Pizzella, A., et al. 2008, MNRAS, 389, 341

Peng, C. Y., Ho, L. C., Impey, C. D., \& Rix, H.-W. 2002, AJ, 124, 266

Prada, F., Gutierrez, C. M., Peletier, R. F., \& McKeith, C. D. 1996, ApJ, 463, L9+

Thomas, D., Maraston, C., \& Bender, R. 2003, MNRAS, 343, 279

Thomas, D., Maraston, C., Bender, R., \& Mendes de Oliveira, C. 2005, ApJ, 621, 673

Vergani, D., Pizzella, A., Corsini, E. M., et al. 2007, A\&GA, 463, 883

Zeilinger, W. W., Vega Beltrán, J. C., Rozas, M., et al. 2001, ApधSSS, 276, 643 